\title{
Colloidal Microworms Propelling via a Cooperative Hydrodynamic Conveyor Belt
}

\author{
Fernando Martinez-Pedrero, ${ }^{1}$ Antonio Ortiz-Ambriz, ${ }^{1}$ Ignacio Pagonabarraga ${ }^{2,3}$ and Pietro Tierno ${ }^{1,3, *}$ \\ ${ }^{1}$ Estructura $i$ Constituents de la Matèria, Universitat de Barcelona, 08028 Barcelona, Spain \\ ${ }^{2}$ Departament de Física Fonamental, Universitat de Barcelona, 08028 Barcelona, Spain \\ ${ }^{3}$ Institut de Nanociència i Nanotecnologia, IN ${ }^{2} U B$, Universitat de Barcelona, 08028 Barcelona, Spain
}

(Received 13 March 2015; published 22 September 2015)

\begin{abstract}
We study propulsion arising from microscopic colloidal rotors dynamically assembled and driven in a viscous fluid upon application of an elliptically polarized rotating magnetic field. Close to a confining plate, the motion of this self-assembled microscopic worm results from the cooperative flow generated by the spinning particles which act as a hydrodynamic "conveyor belt." Chains of rotors propel faster than individual ones, until reaching a saturation speed at distances where induced-flow additivity vanishes. By combining experiments and theoretical arguments, we elucidate the mechanism of motion and fully characterize the propulsion speed in terms of the field parameters.
\end{abstract}

DOI: 10.1103/PhysRevLett.115.138301

PACS numbers: $82.70 . D d, 87.85 . g j$

Propulsion in viscous fluids plays a key role in many different contexts of biology, physics, and chemistry. From a fundamental perspective, the transport of microscopic objects in a liquid medium poses the appealing challenge to find an adequate swimming strategy due to the negligible role of inertial force compared to viscous one. At low Reynolds number the Navier-Stokes equations become time reversible [1], and any strategy based on reciprocal motion, i.e., a motion composed by symmetric backward and forward displacements, will fail to produce net propulsion [2].

Facing this challenge, the last few years have witnessed the theoretical propositions of several suitable geometries and procedures to propel micromachines in viscous fluids [3-9]. Parallel advances in miniaturization have led to the generation of new classes of chemically powered $[10,11]$ or externally actuated [12-15] prototypes with exciting applications in emerging fields such as microsurgery $[16,17]$ or lab-on-a-chip technology $[18,19]$.

In contrast to reaction driven microswimmers, actuated magnetic micropropellers do not present the autonomous behavior that distinguishes force- and torque-free motion of biological organisms [20], but instead avoid efficiency reduction due to fuel shortage or directional randomization. To date, three main approaches have been developed to transport microscopic particles in a viscous fluid using uniform magnetic fields [21], namely, by actuating flexible magnetic tails $[12,22]$, by rotating helical shaped structures $[23,24]$, or by using the close proximity to a bounding wall $[25,26]$. In the latter case, it is well established that in the Stokes regime the rotational motion of a body close to a surface can be rectified into net translation due to the hydrodynamic interaction with the boundary [27]. Surface rotors are optimal to work in confined geometries such as microfluidic channels or biological networks characterized by narrow pores.

In this Letter we show that an ensemble of rotors dynamically self-assembled via attractive dipolar interactions can be propelled by a hydrodynamic "conveyor-belt" effect generated by the cooperative flow of the spinning particles close to a surface. Recent theoretical works [28-30] have addressed the rich dynamics of active rotors lying and spinning in the same plane. Here we rotate our particles perpendicular to the bounding plane and use real time and space experiments to elucidate the mechanism underlying the collective propulsion.

We use monodisperse paramagnetic colloids of radius $a=1.4 \mu \mathrm{m}$, composed by a polymer matrix evenly doped with nanoscale superparamagnetic iron oxide grains (Dynabeads M-270, Invitrogen). The particles are diluted in Millipore water and deposited above a glass substrate where they sediment due to density mismatch. After sedimentation, each particle floats at a distance $h$ from the plate due to the balance between gravity and electrostatic interactions with the negative charged substrate. The particles display small Brownian motion above the substrate $[(x, y)$ plane], with a diffusion coefficient $D=0.14 \mu \mathrm{m}^{2} \mathrm{~s}^{-1}$, and negligible out-of-plane fluctuations.

As shown in the schematic of Fig. 1(a), we have assembled the magnetic particles into translating chains by applying a rotating magnetic field elliptically polarized in the $(x, z)$ plane with angular frequency $\omega, \boldsymbol{H}(t) \equiv$ $H_{x} \cos (\omega t) \boldsymbol{e}_{x}-H_{z} \sin (\omega t) \boldsymbol{e}_{z}$. The amplitude $H_{0}$ and ellipticity $\beta \in[-1,1]$ of the applied field are defined as $H_{0}=$ $\sqrt{\left(H_{x}^{2}+H_{z}^{2}\right) / 2}$ and $\beta=\left(H_{x}^{2}-H_{z}^{2}\right) /\left(H_{x}^{2}+H_{z}^{2}\right)$, respectively. The applied modulation induces a finite magnetic torque on the particles, $\boldsymbol{T}_{m}=\mu_{0}\langle\boldsymbol{m} \times \boldsymbol{H}\rangle$, which sets them in rotation at an angular speed $\Omega$ close to the plane. Here, $\mu_{0}=4 \pi \times 10^{-7} \mathrm{H} \mathrm{m}^{-1}, \boldsymbol{m}$ is the particle magnetic moment, and $\langle\ldots\rangle$ denotes a time average. For high driving frequencies, $\omega \gtrsim 50 \mathrm{rad} \mathrm{s}^{-1}$, the magnetic torque arises due to a finite internal relaxation time of the particle magnetization [31-33] $t_{r}$, which in our case is $t_{r} \sim 10^{-4} \mathrm{~s}$. Upon balancing $\boldsymbol{T}_{m}$ with the viscous torque arising from the rotation in 
(a)
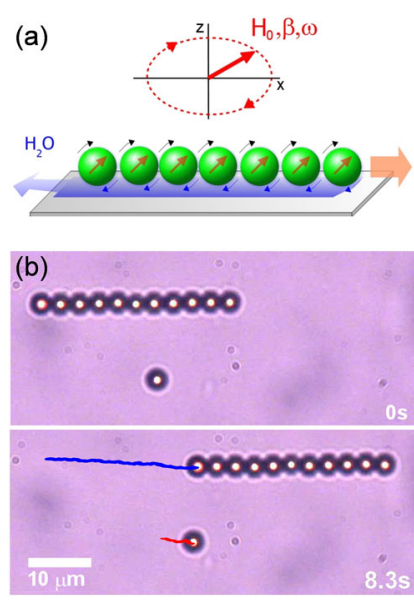

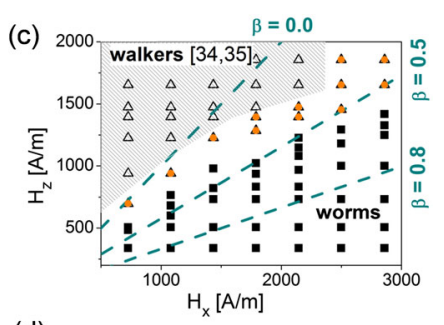

(d)

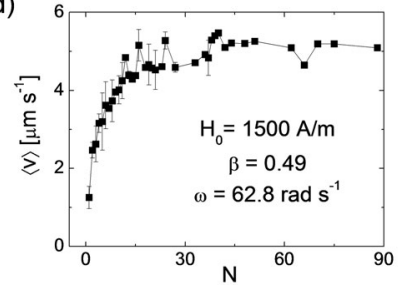

FIG. 1 (color online). (a) Schematic of an ensemble of paramagnetic colloids rotating close to a glass plate and subjected to an elliptically polarized rotating field. (b) Microscope images showing the motion of a chain (blue line) and of an individual rotor (red line) subjected to a rotating field with $H_{0}=1500 \mathrm{~A} \mathrm{~m}^{-1}$, $\beta=0.49$, and $\omega=62.8 \mathrm{rad} \mathrm{s}^{-1}$ at $t=0 \mathrm{~s}$ (top) and $8.3 \mathrm{~s}$ later (bottom). (c) Regions in the $\left(H_{x}, H_{z}\right)$ plane where colloidal "walkers" [43,44] (empty triangles), translating worms (filled squares), or both types of dynamics (filled triangles) are observed $\left(\omega=62.8 \mathrm{rad} \mathrm{s}^{-1}\right)$. (d) Average speed of the colloidal worms $\langle v\rangle$ vs number of particles $N$.

the medium $\boldsymbol{T}_{v}=-8 \pi \eta \Omega a^{3}$ the average rotational speed reads $\langle\Omega\rangle=\mu_{0} H_{0}^{2} \sqrt{1-\beta^{2}} \chi t_{r} \omega / 6 \eta\left(1+t_{r}^{2} \omega^{2}\right)$ [34]. Here $\chi=0.4$ is the magnetic susceptibility under static field and $\eta=10^{-3} \mathrm{Pas}$ denotes the dynamic viscosity of water. Individual rotors translate at a speed $\left\langle\mathbf{v}_{0}\right\rangle \sim\langle\Omega\rangle a \boldsymbol{e}_{x}$ due to the close proximity of the surface which breaks the symmetry. A pair of particles $(i, j)$, a distance $\boldsymbol{r}_{i j}$ away, experience a magnetic dipolar interaction of energy, $U_{d}=\left(\mu_{0} / 4 \pi\right)\left[\left(\boldsymbol{m}_{i} \cdot \boldsymbol{m}_{j}\right) / r_{i j}^{3}-3\left(\boldsymbol{m}_{i} \cdot \boldsymbol{r}_{i j}\right)\left(\boldsymbol{m}_{j} \cdot \boldsymbol{r}_{i j}\right) / r_{i j}^{5}\right]$, which is maximally attractive (repulsive) for particles with magnetic moments parallel (normal) to $\boldsymbol{r}_{i j}$. Performing a time average of the dipolar energy between two colloids for a rotating magnetic field in the $(x, z)$ plane $(\beta=0)$, reveals an effective attractive potential in this plane $\left\langle U_{d}\right\rangle=$ $-\left(\mu_{0} m^{2} / 8 \pi(x+z)^{3}\right)$, leading to chaining along the $x$ direction, while being repulsive in the perpendicular one, $\left\langle U_{d}\right\rangle=\left(\mu_{0} m^{2} / 4 \pi y^{3}\right)$ (similar results holds for $\beta \neq 0$ ). Thus the actuating magnetic field forces the paramagnetic colloids to assemble into elongated chains or "worms," while it also ensures the rotational motion of the individual units.

The dynamics of a colloidal worm is illustrated by the two microscopic images in Fig. 1(b) (VideoS1 in Ref. [34]). Under the conditions of this experiment (caption of Fig. 1), a chain of $N=11$ rotors covers a distance of $\sim 25 \mu \mathrm{m}$ at an average speed of $\langle v\rangle=3.0 \mu \mathrm{m} \mathrm{s}^{-1}$. In the same pair of images, below the traveling chain, one individual rotor is shown covering a smaller distance of $\sim 5.6 \mu \mathrm{m}$ at a lower

speed of $\left\langle v_{0}\right\rangle=0.6 \mu \mathrm{m} \mathrm{s}^{-1}$, making evident the enhanced propulsive behavior of the colloidal worm.

In order to identify the range of field parameters where propelling worms are observed, we perform a series of experiments by varying the two amplitudes of the applied field $\left(H_{x}, H_{z}\right)$, which correspond to values of $\beta \in$ $[-0.73,0.97]$ and $H_{0} \in[600,2400] \mathrm{A} \mathrm{m}^{-1}$, and at a fixed angular frequency of $\omega=62.8 \mathrm{rad} \mathrm{s}^{-1}$. The graph in Fig. 1(c) shows the existence of two distinct types of dynamics: for low ellipticity $(\beta<0.2)$, the chains rotate as a whole, performing a 3D "walkinglike" motion as observed in previous works $[43,44]$. In contrast, for larger values of $\beta>0.45$, where the out of plane component of the field $H_{z} \ll H_{x}$, we observe translating worms. Note that in the intermediate region both types of dynamic states can be found. At fixed frequency (data with different $\omega$ are included in Ref. [34]) walkers can be transformed into worms by increasing the field ellipticity. This effect is caused by the decrease in the azimuthal part of the dipolar force $F_{z} \sim$ $H_{z}\left(\partial H_{z} / \partial z\right)$ due to the corresponding decrease in $H_{z}$, and, hence, the relative vertical displacement of the colloids, in favor of the in-plane part $\left(H_{x}\right)$ of the rotating field during one oscillation period. As a consequence, increasing $\beta$ at constant $\omega$ decreases the oscillations of the phase-lag angle between the main axis of the chain and the rotating field [34]. In the limit case of $\beta=1$ worms still form but the chains do not show a net motion. As shown in Fig. 1(d), at a fixed ellipticity of $\beta=0.49$, and amplitude of $H_{0}=1500 \mathrm{Am}^{-1}$, the average speed $\langle v\rangle$ initially increases with the worm length, and later saturates to a maximum value of $5.3 \mu \mathrm{m} \mathrm{s}^{-1}$. Such a trend of the velocity in terms of the number of rotors is observed both by varying the frequency, or the field amplitude $H_{0}$.

The series of experiments displayed in Fig. 2 serves to elucidate the mechanism underlying the motion of the colloidal worms. Figure 2(a) demonstrates that the rotation of the individual particles within the chain is an essential ingredient for its net displacement. At the top part of Fig. 2(a) a chain of $N=7$ particles is propelled towards the right at an average speed of $\langle v\rangle=2.0 \mu \mathrm{m} \mathrm{s}^{-1}$. The pair of images at the bottom of Fig. 2(a) shows a chain composed by the same number of particles, but where some of them have been previously permanently linked by screening the electrostatic interactions (orange segments) [45]. We observe no net translational motion when subjecting the linked particles to the same field conditions, as shown in VideoS2 in Ref. [34].

Next, in a separate experiment, we analyze the effect of the boundary on the propulsion direction and speed of the colloidal worms. In Fig. 2(b) we compare two chains composed by $N=5$ particles and subjected to the same field conditions, but propelling close to a glass-water interface (images at the top), or to a water-air interface (bottom). The water-air interface was realized by suspending $\sim 0.2 g$ of surfactant-free water in a specially 

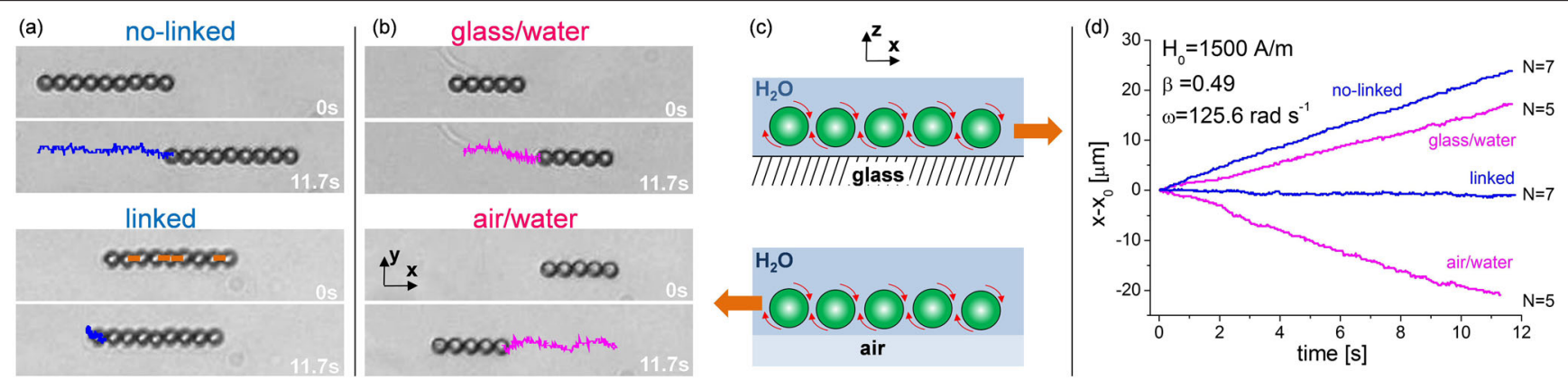

FIG. 2 (color online). (a) Sequence of images showing a worm composed by $N=7$ free rotors (top) or rotors with pairs of particles permanently linked (orange segments, bottom). (b) Propelling chain of $N=5$ rotors at the glass-water interface (top) and at the air-water interface (bottom). Field parameters for (a) and (b) are $H_{0}=1500 \mathrm{~A} \mathrm{~m}^{-1}, \beta=0.49$, and $\omega=125 \mathrm{rad} \mathrm{s}^{-1}$. (c) Schematics illustrating the rotors locations with respect to the interface in (b). (d) Position $\left(x-x_{0}\right)$ versus time for the chains of particles. Black (blue) line refers to (a), gray (pink) to (b).

prepared circular cavity of volume of $0.18 \mathrm{~cm}^{3}$ and provided with a small hole ( $2 \mathrm{~mm}$ diameter) at the bottom. Surface tension is able to hold the deposited droplet while evaporation effects are negligible during the experimental time (see Ref. [34] for details). The paramagnetic colloids sediment approaching the water-air interface. However, as observed previously [46,47], these particles remain in the water phase rather than protruding in the air phase due to their corresponding small contact angle with this interface. As shown in VideoS2 in Ref. [34], close to the water-air interface the chain propels in the opposite direction. Although the interfaces in both cases bind the motion of the colloidal particles and induce a rectification of the rotating colloids, the local flow induced by the stick boundary condition characteristic of the solid wall differs from the one generated by the slip boundary condition imposed by the liquid-air interface, leading to an opposite displacement of the worms. We note that a similar effect has been used in Ref. [48] to reverse the rotation of E. coli. We also find that the average speed of the colloidal worm at the water-air interface $\left(\langle v\rangle=-1.8 \mu \mathrm{m} \mathrm{s}^{-1}\right)$ is $25 \%$ higher than the speed close to the solid surface, $\langle v\rangle=1.5 \mu \mathrm{m} \mathrm{s}^{-1}$.

In order to explain the hydrodynamic origin of the observed rectification effect, we propose a model where the effect of a planar interface on the motion of a solid sphere located at a distance $h$ can be accounted for, to lowest order, through an appropriate set of hydrodynamic singularities at the same distance $h$ below the position of the interface. For a liquid-air interface, the image reduces to a particle rotating in the opposite sense than the actuated colloid, while for a solid surface an additional stresslet and source doublet must be added [49].

A colloid subject to a torque $\boldsymbol{T}$ will rotate and generate a flow field, $\mathbf{v}$, around it that will decay asymptotically as $\mathbf{v}=-\boldsymbol{T} \times \boldsymbol{r} /\left(8 \pi \eta r^{3}\right)$, where $\boldsymbol{r}$ stands for the position vector from the colloid center. In the presence of a liquid interface, the induced flow decays as $\mathbf{v}=-\boldsymbol{T} \times \boldsymbol{r} /\left(8 \pi \eta r^{3}\right)-$ $\boldsymbol{T}^{*} \times \boldsymbol{r}^{*} /\left(8 \pi \eta r^{3}\right)$, where $\boldsymbol{r}^{*}$ is the position vector from the center of the image and $\boldsymbol{T}^{*}=-\eta \boldsymbol{T} / \eta_{g}$, with $\eta_{g}$ being the gas (air) shear viscosity. For a solid interface additional terms must be included, as described in Ref. [34], and $\boldsymbol{T}^{*}=-\boldsymbol{T}$. Thus for a liquid-solid interface the torque acting on the image has the same magnitude that the one actuating on the deposited particle, but for a liquid-air interface the image torque increases by a factor $\eta / \eta_{g}$ [50]. Hydrodynamic rectification emerges from the flow induced by the image singularities because the flow will generally have a component parallel to the interface. As a result, a particle close to an actuated one will be displaced by such induced flow. We can quantify the effect of this displacing flow on an array on $N$ equispaced colloids placed parallel to the wall by computing the velocity at the center of such an assembly $\mathbf{v}_{c}$. If we consider that each colloid moves with the total flow velocity induced by the rest of the rotating colloids at its position (hence assuming it experiences no friction force with respect to the local flow), we can arrive at Ref. [34],

$$
\begin{aligned}
\mathbf{v}_{c}= & v_{0} \boldsymbol{e}_{x}+\frac{\left|v_{0}\right|}{N} \sum_{j=0}^{N} \sum_{i \neq j}\left[1-\xi+\xi\left(\frac{a}{h}\right)^{-2}\right] \\
& \times\left\{\frac{1-\xi}{\left[1+\epsilon^{2}(i-j)^{2}\right]^{3 / 2}}+\frac{3 \xi \epsilon^{2}(i-j)^{2}}{\left[1+\epsilon^{2}(i-j)^{2}\right]^{5 / 2}}\right\} \boldsymbol{e}_{x},
\end{aligned}
$$

which shows that the instantaneous velocity depends on the worm length $N$, and the geometrical parameter $\epsilon \equiv \delta / 2 h$, where $\delta$ is the center-to-center separation between consecutive colloids in the array, while $\xi=1(0)$ for a solid (liquid) substrate, Ref. [34]. Here, $v_{0}$ stands for the rectifying velocity of an isolated colloid placed at a distance $h$ above the liquid-solid interface $v_{0}=T_{0} a^{2} /\left(32 \pi \eta h^{4}\right)$, while $v_{0}=$ $-T_{0} /\left(32 \pi \eta_{g} h^{2}\right)$ for a liquid-gas interface. Therefore, one can understand both the increase in the magnitude of the speed of a worm in the presence of a liquid-gas interface and the change in direction with respect to the solid-liquid interface. Moreover, Eq. (1) illustrates that the difference between interfaces enters through the overall magnitude $v_{0}$ 


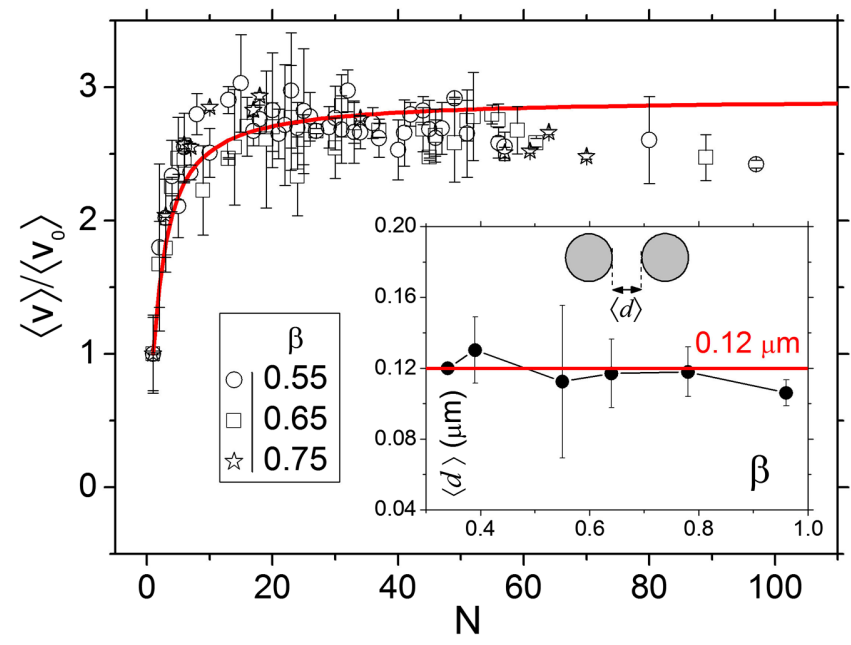

FIG. 3 (color online). Normalized average speed $\langle v\rangle /\left\langle v_{0}\right\rangle$ of colloidal worms as a function of the number of rotors $N$ for different ellipticity $\beta\left(\omega=62.8 \mathrm{rad} \mathrm{s}^{-1}, H_{0}=2300 \mathrm{~A} \mathrm{~m}^{-1}\right)$. Points denote experimental data while the continuous line is a fit following Eq. (1). Inset shows the average surface distance $\langle d\rangle$ vs $\beta$.

and that the remaining dependence in the worm size and elevation from the interface are purely geometrical.

To test the model, we measure the time average velocity $\langle v\rangle$ for a series of worms characterized by different numbers of particles and at different values of $\beta$. Figure 3 shows the comparison between experiment and theory by fitting the rescaled velocity $\langle v\rangle /\left\langle v_{0}\right\rangle$ with Eq. (1). First, the data show that the propulsion speed is almost independent on $\beta$ at parity of actuating amplitude of the applied field. Moreover, we use only one adjustable variable as a fitting parameter, finding $\epsilon=0.92$. From particle tracking we measure the average distance between two particles $\langle\delta\rangle=\langle d\rangle+2 a$, with $\langle d\rangle$ the distance between their surfaces, and confirm that this quantity is indeed independent on $\beta$, and given by $\langle d\rangle=120 \mathrm{~nm}$, as shown in the inset of Fig. 3. Close contact $(\langle d\rangle=0)$ between the particles is prevented by the electrostatic double layer interactions between the particles and steric repulsion due to the polymer coating. The value of $\langle d\rangle$ allows us to find an elevation of the worm from the substrate $h=1.58 \mu \mathrm{m}$ (surface distance from the glass plane $\sim 180 \mathrm{~nm}$ ), similar to the one found by evanescent light scattering of individual particles [51]. The quantitative agreement between experiments and theory, despite the simplifications used in the model [34], demonstrates that the latter well captures all the basic features observed, and provides a clear description of the physical mechanism underlying the worm propulsion.

A moving worm generates a cooperative flow field which continuously advects the colloidal chain in a similar way to a hydrodynamic conveyor belt [52]. This effect is illustrated in Fig. 4, where we plot the normalized average flow velocity $\left\langle v_{f}\right\rangle /\left\langle v_{0}\right\rangle$ produced by a moving worm as a

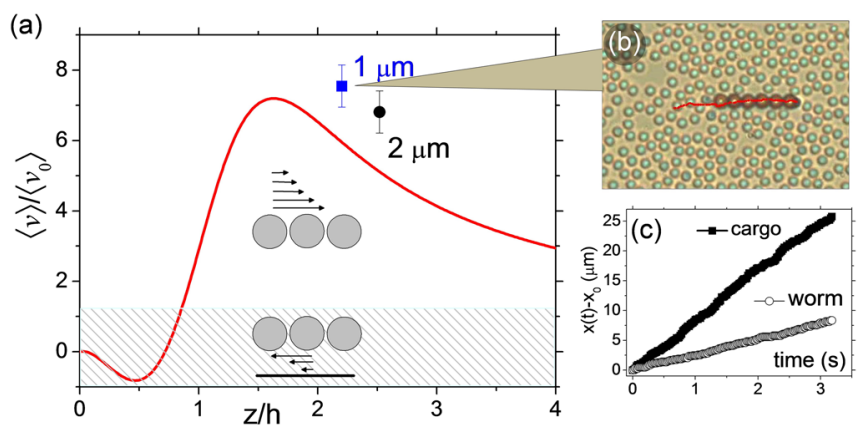

FIG. 4 (color online). (a) Normalized average flow speed $\left\langle v_{f}\right\rangle /\left\langle v_{0}\right\rangle$ vs elevation from the surface $z / h$ and calculated following the model in Ref. [34]. (b) Colloidal worm dragging a $2 \mu \mathrm{m}$ silica particle (red trajectory), VideoS3 in Ref. [34]. (c) Plots of the distance versus time for the worm (empty circles) and silica particle (filled squares).

function of the rescaled elevation $z / h$ (details of the calculation are given in Ref. [34]). In the small region between the worm and the bounding plate the flow velocity is negative, while it changes sign above the moving chain. The flow field can be visualized by tracking the position of nonmagnetic silica particles used as tracers and floating above the plate similar to particle image velocimetry, Fig. 4(b). Once close to the moving chain, the tracers are captured by the hydrodynamic belt and rapidly dragged along the worm till they are expelled at the front, as shown in VideoS3 in Ref. [34]. We confirm this mechanism by measuring the average speed of two different colloidal "cargos" having sizes 1 and $2 \mu \mathrm{m}$ shuffled by the conveyor belt at an average speed of 8.3 and $7.4 \mu \mathrm{m} \mathrm{s}^{-1}$, respectively (here, $v_{0}=1.1 \mu \mathrm{m} \mathrm{s}^{-1}$ ).

In conclusion, we have demonstrated and theoretically supported a concept of directed propulsion at low Reynolds number based on the use of magnetically assembled colloidal rotors. Propulsion arises due to the nonlinear cooperative rectification of flows generated by the spinning particles close to the bounding wall. The hydrodynamic flow generated by our magnetic prototype can be used as an efficient mechanism to transport biological or colloidal cargos entrapped and translated by the conveyor belt [53]. On the other hand, trapping the leading rotor of the worm by optical tweezers could convert the magnetic propeller into a fluid pump which can be readily employed in microand nanofluidc systems.

F. M. P., A. O. A., and P. T. acknowledge support from the ERC starting Grant "DynaMO." P. T. acknowledges support from the "Ramon y Cajal" Program No. RYC2011-07605, from MINECO (FIS2013-41144-P), and DURSI (2014SGR878). I. P. acknowledges support from MINECO (Spain), Project No. FIS2011-22603, DURSI Project No. 2014SGR-922, and Generalitat de Catalunya under Program "ICREA Acadèmia." 
*ptierno@ub.edu

[1] J. Happel and H. Brenner, Low Reynolds Number Hydrodynamics (Noordhoff, Leiden, 1973).

[2] E. M. Purcell, Am. J. Phys. 45, 3 (1977).

[3] H. A. Stone and A. D. T. Samuel, Phys. Rev. Lett. 77, 4102 (1996).

[4] S. Camalet, F. Jülicher, and J. Prost, Phys. Rev. Lett. 82, 1590 (1999).

[5] A. Najafi and R. Golestanian, Phys. Rev. E 69, 062901 (2004).

[6] E. Lauga, Phys. Rev. E 75, 041916 (2007).

[7] G. P. Alexander and J. M. Yeomans, Europhys. Lett. 83, 34006 (2008).

[8] M. T. Downton and H. Stark, J. Phys. Condens. Matter 21, 204101 (2009).

[9] M. Leoni and T. B. Liverpool, Eur. Phys. J. E 35, 126 (2012).

[10] W. F. Paxton, K. C. Kistler, C. C. Olmeda, A. Sen, S. K. S. Angelo, Y. Cao, T. E. Mallouk, P. E. Lammert, and V. H. Crespi, J. Am. Chem. Soc. 126, 13424 (2004).

[11] J. R. Howse, R. A. L. Jones, A. J. Ryan, T. Gough, R. Vafabakhsh, and R. Golestanian, Phys. Rev. Lett. 99, 048102 (2007).

[12] R. Dreyfus, J. Baudry, M. L. Roper, M. Fermigier, H. A. Stone, and J. Bibette, Nature (London) 437, 862 (2005).

[13] A. Snezhko, M. Belkin, I. S. Aranson, and W.-K. Kwok, Phys. Rev. Lett. 102, 118103 (2009).

[14] G. Volpe, I. Buttinoni, D. Vogt, H.-J. Kummerer, and C. Bechinger, Soft Matter 7, 8810 (2011).

[15] A. Bricard, J.-B. Caussin, N. Desreumaux, O. Dauchot, and D. Bartolo, Nature (London) 503, 95 (2013).

[16] B. J. Nelson, I. K. Kaliakatsos, and J. J. Abbott, Annu. Rev. Biomed. Eng. 12, 55 (2010).

[17] K. E. Peyer, L. Zhang, and B. J. Nelson, Nanoscale 5, 1259 (2013).

[18] S. M. H. S. Sanchez, A. A. Solovev, and O. G. Schmidt, J. Am. Chem. Soc. 133, 701 (2011).

[19] J.Wang, Lab on Chip 12, 1944 (2012).

[20] E. Lauga and T. R. Powers, Rep. Prog. Phys. 72, 096601 (2009).

[21] P. Fischer and A. Ghosh, Nanoscale 3, 557 (2011).

[22] O. S. Pak, W. Gao, J. Wang, and E. Lauga, Soft Matter 7, 8169 (2011)

[23] L. Zhang, J. J. Abbott, L. Dong, K. E. Peyer, B. E. Kratochvil, H. Zhang, C. Bergeles, and B. J. Nelson, Nano Lett. 9, 3663 (2009).

[24] A. Ghosh and P. Fischer, Nano Lett. 9, 2243 (2009).

[25] P. Tierno, R. Golestanian, I. Pagonabarraga, and F. Sagués, Phys. Rev. Lett. 101, 218304 (2008).

[26] L. Zhang, T. Petit, Y. Lu, B. E. Kratochvil, K. E. Peyer, R. Pei, J. Lou, and B. J. Nelson, ACS Nano 4, 6228 (2010).

[27] A. J. Goldman, R. G. Cox, and H. Brenner, Chem. Eng. Sci. 22, 637 (1967).

[28] M. Leoni and T. B. Liverpool, Europhys. Lett. 92, 64004 (2010).

[29] Y. Fily, A. Baskaran, and M. C. Marchetti, Soft Matter 8, 3002 (2012).
[30] N. H. P. Nguyen, D. Klotsa, M. Engel, and S. C. Glotzer, Phys. Rev. Lett. 112, 075701 (2014).

[31] P. Tierno, R. Muruganathan, and T. M. Fischer, Phys. Rev. Lett. 98, 028301 (2007).

[32] X. J. A. Janssen, A. J. Schellekens, K. van Ommering, L. J. van Ijzendoorn, and M. J. Prins, Biosens. Bioelectron. 24, 1937 (2009).

[33] A. Cebers and H. Kalis, Eur. Phys. J. E 34, 30 (2011).

[34] See Supplemental Material at http://link.aps.org/ supplemental/10.1103/PhysRevLett.115.138301, which includes Refs. [35-42], more details on the experimental systems, theoretical model used to calculate the rotational dynamics of the chains, the flow field generated and three videoclips.

[35] A. J. Goldman, R. G. Cox, and H. Brenner, Chem. Eng. Sci. 22, 637 (1967).

[36] A. J. Goldman, R. G. Cox, and H. Brenner, Chem. Eng. Sci. 22, 653 (1967).

[37] J. C. Crocker and D. G. Grier, J. Colloid Interface Sci. 179, 298 (1996).

[38] S. Melle, G. G. Fuller, and M. A. Rubio, Phys. Rev. E 61, 4111 (2000).

[39] O. Sandre, J. Browaeys, R. Perzynski, J.-C. Bacri, V. Cabuil, and R. E. Rosensweig, Phys. Rev. E 59, 1736 (1999).

[40] G. Helgesen, P. Pieranski, and A. T. Skjeltorp, Phys. Rev. Lett. 64, 1425 (1990).

[41] G. Helgesen, P. Pieranski, and A. T. Skjeltorp, Phys. Rev. A 42, 7271 (1990).

[42] S. L. Biswal and A. P. Gast, Phys. Rev. E 69, 041406 (2004).

[43] H. Morimoto, T. Ukai, Y. Nagaoka, N. Grobert, and T. Maekawa, Phys. Rev. E 78, 021403 (2008).

[44] C. E. S. L. Schmid, M. F. Schneider, T. Franke, and A. Alexander-Katz, Proc. Natl. Acad. Sci. U.S.A. 107, 535 (2010).

[45] The linkage is achieved by subjecting the pristine particles first to a strong spatially uniform $\left(H \sim 7200 \mathrm{~A} \mathrm{~m}^{-1}\right)$ field in a $100 \mathrm{mM} \mathrm{NaCl}$ solution. The presence of the salt screens the double layer favoring irreversible linkage between the particles via attractive van der Waals forces. Later the linked particles are redispersed in pure water and deposited on top of the glass substrate.

[46] L. E. Helseth, T. H. Johansen, and T. M. Fischer, Appl. Phys. Lett. 93, 042516 (2008).

[47] F. Ebert, P. Dillmann, G. Maret, and P. Keim, Rev. Sci. Instrum. 80, 083902 (2009).

[48] R. DiLeonardo, D. DellArciprete, L. Angelani, and V. Iebba, Phys. Rev. Lett. 106, 038101 (2011).

[49] J. R. Blake and A. T. Chwang, J. Eng. Math. 8, 23 (1974).

[50] I. Pagonabarraga, M. H. J. Hagen, C. P. Lowe, and D. Frenkel, Phys. Rev. E 58, 7288 (1998).

[51] V. Blickle, D. Babic, and C. Bechinger, Appl. Phys. Lett. 87, 101102 (2005).

[52] B. U. Felderhof, J. Chem. Phys. 136, 164905 (2012).

[53] F. Martinez-Pedrero and P. Tierno, Phys. Rev. Applied 3, 051003 (2015). 